\title{
Über ból und symptomy... Wortschatz im Polnischunterricht für deutschsprachige Rettungskräfte
}

\author{
About ból and symptomy... \\ Lexical analysis in Polish as a foreign language classes \\ for German paramedics
}

\begin{abstract}
This paper focuses on the lexical side of the emergency communication of German paramedics who are learning Polish as a foreign language for professional purposes. The aim of the study is to find out which lexical units arise during language courses and how often. To what extent can the acquired vocabulary be used to develop dedicated programmes for foreign language acquisition in medical emergency communication? The analysis shows that the vocabulary collected here depends on the subject, such as ból (pain) or leki (medicines). According to the participants, symptomy (symptoms) and duszności (shortness of breath) are also part of the common vocabulary. Emergency medical communication can be carried out with the question pronouns kiedy (when) and gdzie (where). Among the most frequently repeated lexical units of vital parameters during patient transfer are saturacja (oxygen saturation) and puls (pulse). The vocabulary collected here should help in preparing a description of the competences which should form part of a language course for professional purposes.
\end{abstract}

KEYWORDS: Emergency medical communication, Polish as a foreign language, vocabulary.

SCHLÜsSELWÖRTER: Medizinische Notfallkommunikation, Polnisch als Fremdsprache, Wortschatz.

Fremdsprachendidaktiker und Linguisten ${ }^{1}$ wissen, wie wichtig eine geglückte Zusammenstellung der Inhalte eines Fremdsprachenkurses für berufliche Zwecke ist. In der Forschungsgemeinschaft werden Stimmen laut, die nach einer einheitlichen Beschreibung der Fertigkeiten bei Sprachkursen für berufliche

\footnotetext{
${ }^{1}$ Aus Gründen der besseren Lesbarkeit wird im Text verallgemeinernd das generische Maskulinum verwendet. Diese Formulierungen umfassen gleichermaßen weibliche, männliche oder diverse Personen. Alle sind damit gleichermaßen angesprochen.
} 
Zwecke plädieren (Gajewska 2011: 19). Die Wortschatztiefe und breite spielt bei einer solchen Beschreibung eine wichtige Rolle. Erste bedeutende Schritte in diese Richtung wurden bereits getan (Gębal \& Kołsut 2019: 67), wobei die notfallmedizinische Kommunikation auf Polnisch noch Aufarbeitung bedarf.

Dieser Artikel thematisiert daher das Programm des Polnischunterrichts für Rettungskräfte (Ärzte und Notfallsanitäter) im Rahmen des aus den Mitteln des Fonds für Regionale Entwicklung (EFRE) finanzierten Projekts InGRiP ${ }^{2}$. Im Fokus des Beitrags steht der Wortschatz im Fremdsprachenunterricht für deutschsprachige Rettungskräfte. Unabhängig von dem Sprachkurstyp weisen Forscher im Kontext der Fremdsprachendidaktik auf die Rolle des Wortschatzes hin: „Wortschatzkenntnisse, die nicht nur zum Verstehen fremdsprachlicher Kommunikate, sondern auch zum korrekten Gebrauch der lexikalischen Einheiten [...] und zur Realisierung der kommunikativen Ziele unentbehrlich sind, spielen eine große Rolle im Fremdsprachenlernprozess" (Targońska 2014: 171).

Da Gespräche mit Patienten in einer vor kurzem gelernten Sprache (hier: Polnisch) herausfordernd sind und ein Zusammenspiel der Fertigkeiten wie Hören und Sprechen darstellen, besteht in der Beschreibung der Fertigkeiten mit dem dazugehörigen Wortschatz ein besonderer Mehrwert für diesen Text. Dabei konzentriert sich die vorliegende Untersuchung auf Frequenzerscheinungen, um nach möglichen Rückschlüssen für weitere Sprachkurse und Programme für Fremdsprachenerwerb zu suchen. Dazu wird auf Daten aus dem Projekt InGRiP zurückgegriffen.

Mit Blick auf notfallmedizinische Kommunikation sowie gezielter Wortschatzerwerb im Fremdsprachenunterricht stellen sich folgende Fragen: Welche lexikalischen Einheiten kommen während der Sprachkurse für berufliche Zwecke wie häufig vor? Inwiefern kann der erfasste Wortschatz genutzt werden, zielgerichtete Programme für den Fremdspracherwerb in der medizinischen Notfallkommunikation zu entwickeln? ${ }^{3}$

Aus so konzipierter Fragestellung resultiert die Gliederung des Textes: Im ersten Teil wird das Projekt vorgestellt und auf die Spezifika notfallmedizinischer Kommunikation kurz eingegangen. Darüber hinaus werden das Lehrkonzept sowie die Bedeutung der Lexik im Fachsprachenunterricht nachgezeichnet. Im Hauptteil des Beitrags werden die Daten und das Forschungsdesign vorge-

${ }^{2}$ Nähere Informationen zum Projekt Integrierter grenzüberschreitender Rettungsdienst Pomerania / Brandenburg (InGRiP), das in den Jahren 2018-2021 realisiert wird, sowie zu den beteiligten Partnern finden Sie unter https://www.medizin.uni-greifswald.de/ingrip/de/home/ [Zugriff am: 20.08.2020].

${ }^{3}$ Die hier auf Lehrprogramme ausgerichtete Untersuchung resultiert aus der Multiperspektivität der Begrifflichkeit Fremdsprachendidaktik, die hier sowohl im Sinne von Fluck (vgl. 1992: 16) als auch von Grucza (vgl. 2010: 42) verstanden wird. 
stellt, um dann die Forschungsfragen aufzugreifen und anhand des gesammelten Materials zu beantworten. Im letzten Teil des Artikels werden die Ergebnisse zusammengefasst und mit einem Ausblick versehen.

\section{DAS PROJEKT INGRIP UND DIE NOTFALLMEDIZINISCHE KOMMUNIKATION}

Das Projekt InGRiP ist ein multiinstitutionelles und grenzüberschreitendes Vorhaben, dessen fremdsprachenbezogene Module am Institut für Slawistik der Universität Greifswald verankert wurde. Im Projekt geht es um die transnationale notfallmedizinische Versorgung der Patienten im deutschpolnischen Grenzraum. Somit gehören die fremdsprachliche Kommunikation mit den Patienten sowie die Arbeit in mehrsprachigen Notfallteams zu den zentralen Themen des Vorhabens. Daher wurden berufsbezogene Sprachschulungen sowohl auf Polnisch (für deutsche Rettungskräfte) als auch auf Deutsch (für ihre polnischen Kollegen) konzipiert und Lehr und Lernmaterialien entwickelt ${ }^{4}$. Des Weiteren nahmen Rettungskräfte aus beiden Ländern gemeinsam an einem abschließenden Simulationstraining teil. Dabei lag der Schwerpunkt auf der Erfassung und Auswertung von Problemen in der deutschpolnischen notfallmedizinischen Kommunikation ${ }^{5}$.

Die im Projekt untersuchte Notfallkommunikation gehört zur medizinischen FacetoFace Kommunikation, an der Patienten und ein Rettungsteam beteiligt sind. Darüber hinaus können bei der Kommunikation Familienangehörige oder Unfallzeugen vorkommen. Nicht nur die Rollenwahrnehmung, sondern auch der Erwerb elementarer kommunikativer Äußerungsmuster auf Polnisch sind im fremdsprachigen Kommunikationsgefüge von Bedeutung. Rettungsschemata, die für die Notfallmedizin typisch sind, beinhalten die elementaren Äußerungsmuster und können somit produktiv im Fremdsprachenunterricht genutzt werden. Die medizinischen Schemata, die während der Notfallanamnese angewandt werden und vor allem auf Akronymen ${ }^{6}$ aufbauen, können nicht nur für eine schnelle Übersicht sorgen, sondern auch für Sachlichkeit und Genauigkeit (Stemmler \& Hecker 2017: 100), die das A und O gelungener notfallmedizinischen

\footnotetext{
${ }^{4}$ In der weiteren Beschreibung des Schulungskonzepts sowie bei der Datensammlung wird nur auf Polnisch als Fremdsprache eingegangen.

${ }^{5}$ Die Begriffe notfallmedizinische Kommunikation und Notfallkommunikation werden hier synonym verwendet.

${ }^{6}$ Mit Schemata der Notfallanamnese, die als Akronyme vorkommen, sind Fragehandlungen gemeint, dank derer gezielt Informationen eingeholt werden können. Mehr zu den konkreten Schemata finden Sie bei Stemmler und Hecker (2017).
} 
Kommunikation, auch auf Polnisch, sind. Deswegen lassen sich die Schemata gut im Rahmen der kommunikativen oder Aufgaben orientierten Methode auch als ChunkingSequenzen ${ }^{7}$ realisieren, insbesondere als fertige dialogische notfallmedizinische FrageAntwortSequenzen.

Da sich notfallmedizinische Kommunikation aus konkreten Sprechhandlungen zusammensetzt, die in verschiedene Sprechakte gegliedert werden (Searle 1997: 39), spielen assertive, direktive, erotetische und expressive Sprechakte und die damit verbundenen Fragehandlungen ${ }^{8}$ auch im Unterricht eine wichtige Rolle (Lisek 2020: 193). Um sowohl die ChunkingSequenzen als auch spontane Äußerungen produzieren zu können, benötigen die Teilnehmenden Wortschatzkenntnisse. Wobei nach Targońska (2011: 117) hervorzuheben ist:

Wortschatzkenntnisse bilden nur eine Grundlage für die Fähigkeit, mit dem Wortschatz richtig umzugehen. Seit der kommunikativen Wende ist klar, dass beim Fremdsprachenlernen nicht Sprachwissen, sondern Sprachfertigkeiten bzw. Kompetenzen eine viel wichtigere Rolle spielen.

Mehr zu den Herausforderungen in Bezug auf den Wortschatzerwerb und seine Stellung in Kursen für berufliche Zwecke findet sich im nächsten Kapitel.

\section{FREMDSPRACHENUNTERRICHT FÜR BERUFLICHE ZWECKE UND LEHRKONZEPTE FÜR DIE NOTFALLMEDIZINISCHE KOMMUNIKATION AUF POLNISCH}

Der folgende Abschnitt wird in zwei Unterkapitel gegliedert, um einzeln auf die Schwerpunkte einzugehen: allgemein auf den Fremdsprachenunterricht für berufliche Zwecke für Polnisch und speziell auf die Rolle des Wortschatzerwerbs ${ }^{9}$. Darüber hinaus wird die Konzeption des Unterrichts thematisiert.

\footnotetext{
${ }^{7}$ Chunking ist in Anlehnung an Roche (vgl. 2013: 116) als automatisierte Wiedergabe von festen Formeln oder Redewendungen zu verstehen.

${ }^{8}$ Diese sind dahingehend unterrichtsrelevant, dass bei der Notfallkommunikation beispielsweise konkrete Beschwerden thematisiert werden.

${ }^{9}$ Es wird hier bewusst von Wortschatz und nicht von Vokabeln oder Lexemen geschrieben. Wortschatz wird hier als eine Gesamtheit verstanden, als ein Netz von Wechselwirkungen der Wortarten, die nicht separat im Lernprozess zu betrachten sind. Jegliche Ausdifferenzierungen oder Darstellung, die die Wortarten oder einzelne Wortartgruppen fokussieren, dienen einer Veranschaulichung der Inhalte und stellen dann Wortschatz auf eine vereinfachte Art und Weise dar, was jedoch nicht den Sinn der Untersuchung in Frage stellt, da durch den Wortschatz eine umfängliche Beschreibung der Sprachkompetenzen ermöglicht wird. Daher wird hier neben Wortschatz auch die Bezeichnung lexikalische Einheit benutzt, die nicht mit Lexem gleichzusetzen ist.
} 


\subsection{Fremdsprachenunterricht und Wortschatz für berufliche Zwecke}

Am Anfang des Unterkapitels wird die in der polonistischen Fremdsprachendidaktik in Polen dominierende Differenzierung der Sprachkurse in Kurse für akademische Zwecke und Kurse für berufliche Zwecke (Zarzycka 2017: 137; Seretny 2017: 150) in den Vordergrund gestellt. Mit dieser Perspektive fällt es wesentlich leichter, den Status des Wortschatzes in den Fremdsprachenkursen für deutschsprachige Rettungskräfte einzuordnen. Kurse für akademische Zwecke haben nämlich einen eher allgemeinen Charakter und richten sich an angehende Experten und nicht an einen Personenkreis der bereits beruflich tätigen. Dabei ist auch die Stellung des Wortschatzes dahingehend zu den Kursen für berufliche Zwecke unterschiedlich, dass Kurse für akademische Zwecke prinzipiell nicht nur alle Fertigkeiten fokussieren, sondern auch alle systemlinguistisch relevanten Bereiche didaktisch aufgreifen, d.h. der Stellenwert der Aufarbeitung von grammatischen Phänomenen ist der Wortschatzarbeit gleich. Bei den Kursen für berufliche Zwecke muss es nicht der Fall sein, da auch in der Regel bereits die systemsprachliche Kompetenz vorliegt.

Der Sprachkurs für Rettungskräfte aus den Rettungswachen in den Landkreisen VorpommernGreifswald und MärkischOderland betrifft einen sehr komplexen Bereich der Medizin, nämlich die Notfallmedizin. Die Vorbereitung eines solchen Kurses ist, wie Gębka-Wolak (2011:39) zurecht über Sprachkurse für berufliche Zwecke behauptet, wie eine Einzelanfertigung. In diesem Fall ist es vor allem Pionierarbeit, da bislang ein Sprachkurs speziell für deutsche Rettungskräfte weder konzipiert noch durchgeführt wurde. Daher existieren auch keine profilierten notfallmedizinischen Lehrmaterialien für das Polnische. Diese sind eher für das Sprachniveau B1 typisch, worauf Gębka-Wolak (2016: 11) hinweist ${ }^{10}$.

Ein besonderes Augenmerk gilt auch den betroffenen Berufsgruppen: Laut Seretny (2017: 157) ist es wichtig, zwischen berufserfahrenen Teilnehmenden und erst in den Beruf einsteigenden Absolventen zu differenzieren. An den Sprachkursen im InGRiPProjekt nahmen tatsächlich Rettungskräfte mit 20jähriger Erfahrung im Beruf sowie Rettungssanitäter gleich nach der Berufsschule teil. Die Teilnehmenden verband die Tatsache, dass die Mehrheit noch nie Polnisch gelernt hat, was eine verkürzte Bedarfsanalyse aus dem Jahr

${ }^{10}$ Es existierten zwar Lehrbücher, die medizinische Themen betreffen (z. B. „Co Panu dolega?" von Chłopicka-Wielgos, Pukas-Palimąka \& Turek-Fornelska 2015) oder sich explizit dem Rettungswesen (z. B. Dwujęzyczny podręcznik komunikacji językowej dla stużb ratowniczych pogranicza polskosłowackiego von Majewska-Wójcik, Račáková, Smoleń-Wawrzusiszyn \& Olchowa 2016) widmen, jedoch keine von denen ist für medizinische Rettungskräfte explizit gedacht und somit nicht auf sprachliche notfallmedizinische Vorgänge fokussiert. 
2018 zeigte: „Die Befragten meinten bei einer weiteren Frage bezüglich des Polnischen, zu ca. 97\%, diese Sprache nicht zu beherrschen“ (Lisek 2019: 22). Dies erleichterte nicht unbedingt die Zusammensetzung der Inhalte für den Sprachkurs und insbesondere nicht die Wahl des Wortschatzes, zumal diese prinzipiell als schwierig in der Fachliteratur (Seretny 2017: 159) eingestuft wird. Zugleich wird darauf hingewiesen, dass von dem Bekanntheitsgrad des Wortschatzes der Kommunikationserfolg unter den Lernenden abhängig ist (Seretny 2010: 547).

Dabei ist der Standpunkt von Kósa (2019:32) zu berücksichtigen: „Eines der Hauptziele des Fremdsprachenunterrichts ist die Entfaltung der lexikalischen Kompetenz, weil die Schüler die Lexeme nicht nur verstehen müssen, sondern sie sollten im Stande sein die gelernten Wörter zielgerecht und möglichst geläufig anzuwenden". Somit tritt der produktive Wortschatz in den Vordergrund. Dies geschieht zurecht, denn, wie Kósa (2019: 32) weiter ausführt, der Wortschatz sowohl für rezeptive als auch produktive Fertigkeiten eine Bedeutung hat. Der hier thematisierte Fremdsprachenkurs fokussiert sowohl die produktive Fertigkeit (Sprechen) als auch die rezeptive Fertigkeit (Hören). Erfolgreiche Umsetzung beider hängt mit dem Wortschatzerwerb zusammen.

\subsection{Lehrkonzept für notfallmedizinisches Polnisch im InGRiPProjekt}

Die auf drei Wochen verteilten Lernmodule sind dem Hauptziel des Projekts untergeordnet, also „den Teilnehmenden effiziente Strategien der Kommunikation in der Fach und Nachbarsprache Polnisch beizubringen“ (Lisek 2019: 23). Demzufolge wurde der Unterricht der jeweils vier Gruppen strukturiert und die Inhalte auf Präsenzveranstaltungen und ELearningPhasen verteilt. Die Präsenzveranstaltungen dauerten jeweils eine Woche pro Modul und wurden über das Jahr 2019 verteilt ${ }^{11}$ (Januar bis April sowie August bis Oktober) und behandelten folgende Schwerpunkte: (1) sprachliche Strukturen des Polnischen, (2) medizinische Kommunikation sowie (3) notfallmedizinische Sprechhandlungen in der Fremdsprache. Um eine kontinuierliche und nachhaltige Beschäftigung der Kursteilnehmenden mit der polnischen Sprache zu ermöglichen, wurden Elemente des BlendedLearnings in das Schulungsprogramm integriert. Es wurden

${ }^{11}$ Die Corona-Pandemie hat eine bedeutende Auswirkung auf die Realisierung des Projekts. Außer den geplanten Online-Modulen wurden auch Auffrischungskurse konzipiert und seit Dezember 2019 vor den jeweiligen Simulationstrainings (teils als Präsenzveranstaltung, teils als Online-Veranstaltung) durchgeführt. 
ELearningModule vorgesehen, die in der Zeit zwischen den Präsenzschulungen und vor den Simulationstrainings von den Kursteilnehmenden selbstständig bearbeitet werden konnten ${ }^{12}$.

\section{DATEN UND FORSCHUNGSDESIGN}

Um die Frequenz des Vorkommens notfallmedizinisch relevanten Wortschatzes deutschsprachiger Rettungskräfte im Polnischunterricht analysieren zu können, wurden Sprachdaten gesammelt. Die vorliegenden Sprachdaten wurden im Rahmen einer schriftlichen Aufgabe erhoben, die als Test mit offener Frage konzipiert und während der dritten Woche des Polnischunterrichts zwischen August und Oktober 2019 in Greifswald realisiert wurde.

Für die Umsetzung der Aufgabe im Unterricht wurden drei thematische Bereiche ausgemacht: notfallmedizinische Anamnese, Schmerzuntersuchung sowie Patientenübergabe. Die Wahl resultiert aus der alltagspraktischen Relevanz der Sprechhandlungen für die Teilnehmenden, d. h. im Fokus stehen die den Rettungskräften wichtigen Situationen, in denen die fremdsprachliche Kompetenz insbesondere zur Geltung kommt.

Für alle drei Themenbereiche sollten die Kursteilnehmenden vor der Ausstrahlung eines Lehrfilmes bis zu zehn Worte ${ }^{13}$ auf das Aufgabenblatt ${ }^{14}$ notieren. Die Lehrperson gewährte den Probanden eine Bearbeitungszeit von maximal fünf Minuten. Die Aufgabe sollte ohne Hilfsmittel als Einzelarbeit realisiert werden.

Die folgende Abbildung ist ein beispielhafter Beleg zum Themenbereich „Patientenübergabe“ und zeigt, wie die Teilnehmenden die Aufgabe verstanden haben: Zu erkennen ist folgender Wortschatz: drogi oddechowe (Atemwege), ból (Schmerz), puls (Puls), saturacja (Sauerstoffsättigung), podaliśmy leki (wir haben Medikamente verabreicht), szmer oddechowy (Atmungsgheräusch), niezagrożone (nicht gefährdet), przytomnie (bewusst).

${ }^{12}$ Eine ausführliche Beschreibung der Präsenz- und Online-Module sowie des Kursverlaufs finden Sie u. a. bei Lisek (2018) oder Hryniewicz und Lisek (2019).

${ }^{13}$ Die Bezeichnung Wort wurde aus forschungspraktischen Gründen als ein Kompromiss auf dem Arbeitsblatt für Teilnehmende benutzt, um den Teilnehmenden einen guten Zugang zum Test zu erleichtern und eine zeitschonende Durchführung der Untersuchung zu gewährleisten.

${ }^{14}$ Die Aufgabenstellung war für alle Themenbereiche gleich formuliert und hat sich nur durch die Benennung des Themenbereichs unterschieden und lautete beispielsweise für "notfallmedizinische Anamnese“ wie folgt: „Sie sehen gleich einen Film zum Thema SAMPLE(R). Ohne den Film gesehen zu haben, welche Worte erwarten Sie in der Aufnahme zu hören." 


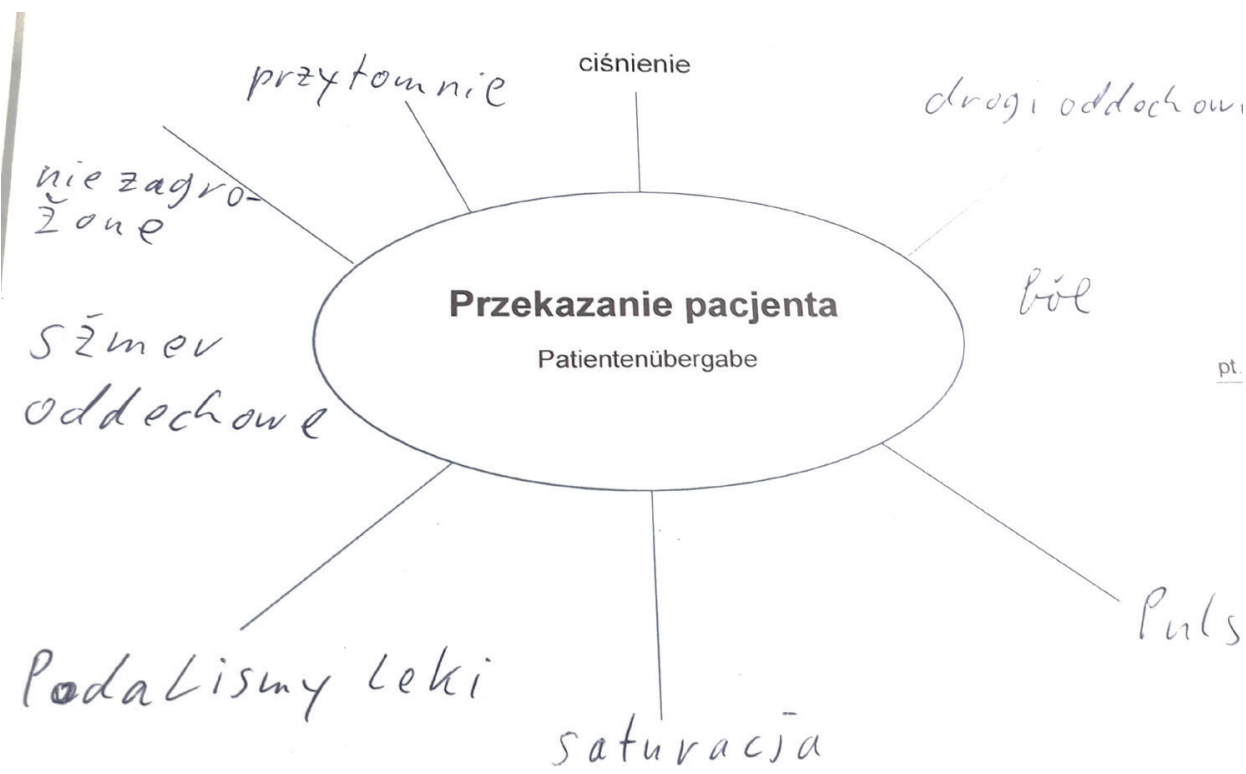

Abbildung 1. Beispielhafte Lösung der Aufgabe aus dem Themenbereich „Patientenübergabe“

Bei der Methode der Auswertung des Datenmaterials handelt es sich um ein quantitatives Verfahren (Albert \& Marx 2016: 12), das typisch für die traditionelle und theoretische Linguistik ist. Es wird nach Häufigkeiten im Wortschatz gesucht, um daraus Regularitäten für zukünftige Sprachkursprogramme abzuleiten.

Als Grundlage der Untersuchung fungieren auch orthografisch falsch dargestellte Lexeme, solange diese das korrekt geschriebene Wort erkennen lassen.

Zum Themenbereich „notfallmedizinische Anamnese“ wurden 38 Einheiten ausgemacht, die sowohl einzeln als auch als Phrasen vorkommen. Kursteilnehmende haben im zweiten Themenbereich "Schmerzuntersuchung“ 43 lexikalische Einheiten genannt. Zum letzten Themenbereich also „Patientenübergabe“ wurden 43 Einheiten festgestellt. Insgesamt wurden in den drei Themenbereichen 113 lexikalische Einheiten gesammelt. Diese wurden erneut auf Wiederholungen überprüft, was eine endgültige Anzahl von 93 Untersuchungseinheiten ergab. Das so entstandene Korpus kann nach Bedarf sowohl nach Wortarten als auch nach thematischen Kategorien gruppiert werden.

Der Abbildung ist zu entnehmen, dass Substantive zusammen mit Pronomen in der gesammelten Sprachdatenprobe dominieren, sie machen nämlich 58\% aus. Die zweitgrößte Gruppe lexikalischer Einheiten repräsentieren Verben (12\%). Der dritte Platz gehört den Adjektiven, die 11\% des gesammelten Datenmaterials 
bilden. Die hier präsentierte Verteilung ${ }^{15}$ überrascht nicht, wenn angenommen wird, dass ein Fremdsprachenkurs für berufliche Zwecke eine hohe nominale Last hat.

Verteilung der erfassten lexikalischen Einheiten nach Wortarten

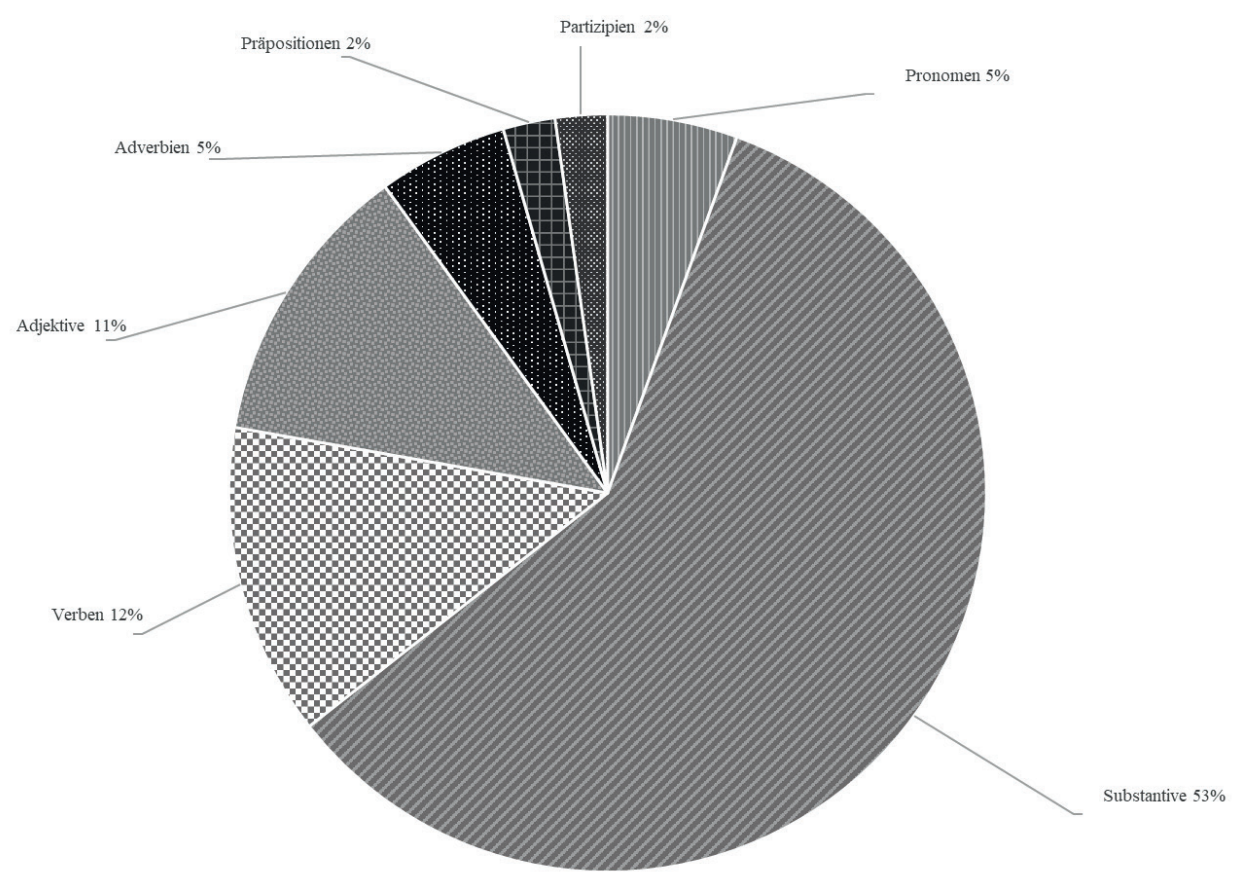

Abbildung 2. Gesammelte lexikalische Einheiten nach Wortarten

\section{ANALYSE DES WORTSCHATZES}

Die Untersuchung richtet sich nach den drei festgelegten Themenbereichen. Es wird der während des Tests gesammelte Wortschatz auf seine Häufigkeit analysiert. Aus forschungspraktischen Gründen werden in der Auswertung drei sich am häufigsten wiederholende lexikalische Einheiten thematisiert. Der Vergleich des gesammelten Wortschatzes zwischen den Gruppen erfolgt nicht

${ }^{15}$ Da unter den Wortarten auch einzelne Präpositionen oder Partizipien vorgekommen sind, wird auf die exemplarisch eingegangen. $\mathrm{Zu}$ den Partizipien gehörte vor allem promieniujący (ausstrahlend). Die Präpositionen kamen vor allem in Phrasen, wie z. B. od kiedy (seit wann) oder od 0 do 10 (von 0 bis 10). 
in Bezug auf die Häufigkeit der ausgemachten Einheiten, sondern auf das Vorkommen einer konkreten lexikalischen Einheit überhaupt ${ }^{16}$.

\subsection{Wortschatz im Themenbereich „notfallmedizinische Anamnese"}

Allgemein ist festzuhalten, dass im Bereich der notfallmedizinischen Anamnese fast 40 sich nicht wiederholende lexikalische Einheiten ausgemacht wurden. Die Frequenzanalyse ergab, dass Folgendes am häufigsten genannt wurde:

Tabelle 1. Am häufigsten gennannte lexikalische Einheiten im Themenbereich „notfallmedizinischen Anamnese"

\begin{tabular}{|l|c|l|c|}
\hline \multicolumn{1}{|c|}{ Lexikalische Einheit } & Häufigkeit & \multicolumn{1}{c|}{ Lexikalische Einheit } & Häufigkeit \\
\hline ból (Schmerz) & 14 & co (was) & 4 \\
\hline leki (Medikamente) & 14 & duszności (Atemnot) & 4 \\
\hline kiedy (wann) & 5 & symptomy (Symptome) & 4 \\
\hline
\end{tabular}

Ból und leki sind somit lexikalische Einheiten, die von der Frequenz her am häufigsten vorkommen. Des Weiteren gehört zu den von den Kursteilnehmenden häufig genannten Einheiten auch kiedy. In allen vier Gruppen kommen sowohl ból als auch leki vor. Auch kiedy wird von den Teilnehmenden aller Gruppen genannt.

Im gesamten Themenbereich setzt sich der ausgemachte Wortschatz anhand von Wortarten wie folgt zusammen: Fast zu 60\% überwiegen Substantive, gefolgt von Pronomen (21\%). Verben machen nur 13\% des hiesigen Wortschatzes aus. Mit $6 \%$ sind Adverbien am wenigsten repräsentiert. Eine detaillierte $\mathrm{Zu}-$ sammensetzung des Wortschatzes nach Wortarten ist der Tabelle zu entnehmen.

Tabelle 2. Wortschatz zum Thema „notfallmedizinische Anamnese“ nach Wortarten ( $\mathrm{n}=38)$

\begin{tabular}{|c|c|c|c|c|}
\hline Wortart & Adverb & Pronomen & Substantiv & Verb \\
\hline Anzahl & 3 & 8 & 22 & 5 \\
\hline
\end{tabular}

Um die Realisierung weiterer notfallmedizinischen Sprachkurse des Polnischen für berufliche Zwecke zu ermöglichen, dient der substantivische Wortschatz als Exemplifikation der unter den Wortarten am zahlreichsten vorkommenden lexikalischen Einheiten.

${ }^{16}$ Dieses Vorgehen ist der Situation geschuldet, dass die vier Gruppen in der letzten Kursphase eine unterschiedliche Anzahl an Teilnehmenden aufweisen. Darüber hinaus erfolgte der Sprachunterricht in der zweiten Gruppe in Form von Einzelunterricht, somit sind mögliche Vergleiche bezüglich der Frequenz der lexikalischen Einheiten zwischen den Gruppen hinfällig. 
Zu der Kategorie gehört folgender Wortschatz: alergia (Allergie), astma (Asthma), ból (Schmerz), choroba (Krankheit), cukrzyca (Diabetes), duszności (Atemnot), głowa (Kopf), goraczka (Fieber), kaszel (Husten), klatka piersiowa (Brustkorb), krew (Blut), lat ${ }^{17}$ (Jahre), leki (Medikamente), nadciśnienie (Bluthochdruck), operacja (Operation), pan (Herr / Sie), serce (Herz), symptomy (Symptome), szpital (Krankenhaus), temperatura (Temperatur), wymioty (Erbrechen), zawat (Herzinfarkt).

Auch wenn Verben nicht zu den am stärksten repräsentierten Gruppen gehören, werden hier die ausgemachten Einheiten beispielhaft genannt: boli, dolega, jadła, ma, stało się. Um Sprachkurse für notfallmedizinische Kommunikation realisieren zu können, werden die Verben in Infinitivform erneut herangezogen, d.h. von den Teilnehmenden wurden folgende Einheiten aufgelistet: boleć (schmerzen, wehtun), dolegać (fehlen), jeść (essen), mieć (haben), stać się (passieren, sich ereignen).

\subsection{Wortschatz im Themenbereich "Schmerzuntersuchung“}

Im Bereich der Schmerzuntersuchung konnten 43 sich nicht wiederholende Einheiten ausgemacht werden. Am häufigsten wurden von den Teilnehmenden, wie es der Tabelle zu entnehmen ist, folgende lexikalische Einheiten genannt:

Tabelle 3. Am häufigsten gennannte lexikalische Einheiten im Themenbereich "Schmerzuntersuchung“

\begin{tabular}{|l|c|}
\hline \multicolumn{1}{|c|}{ Lexem } & Häufigkeit \\
\hline kiedy (wann) & 12 \\
\hline gdzie (wo) & 10 \\
\hline od (seit) & 9 \\
\hline
\end{tabular}

Die Tabelle zeigt, dass Fragehandlungen in diesem thematischen Bereich einen sehr hohen Stellenwert haben. Dies ist insbesondere sichtbar durch die Frequenz von kiedy und gdzie. Die Gruppen vergleichend kommt kiedy nur in drei Gruppen vor, dagegen $g d z i e$ wird von Teilnehmenden in allen vier Gruppen genannt.

Die hier aufgeführten Einheiten - Pronomen (19\%) und Präposition (7\%) dienen vor allem der zeitlichen und räumlichen Schmerzbestimmung. Sie reprä-

${ }^{17}$ Da bei den anderen Einheiten des Wortschatzes diese mehrheitlich in Nominativ vorkommen, ist es mir ein Anliegen die Konstruktion Wie alt sind Sie?, also Ile ma pan(i) lat?, zu thematisiern, da lat hier genitivisch nachgestellt als Substantiv benutzt wird, was im Deutschen adjektivisch realisiert wird. 
sentieren nicht die größte Wortartgruppe, d.h. Substantive (38\%). Sie werden aber gleich nach diesen und noch vor den Verben (14\%) aufgelistet, was der unteren Zusammenstellung zu entnehmen ist.

Tabelle 4. Wortschatz zum Thema „Schmerzuntersuchung“ nach Wortarten $(n=43)$

\begin{tabular}{|c|c|c|c|c|c|c|c|}
\hline Wortart & Adjektiv & Adverb & Partizip & $\begin{array}{c}\text { Präposi- } \\
\text { tion }\end{array}$ & $\begin{array}{c}\text { Pro- } \\
\text { nomen }\end{array}$ & $\begin{array}{c}\text { Substan- } \\
\text { tiv }\end{array}$ & Verb \\
\hline Anzahl & 6 & 2 & 2 & 3 & 8 & 16 & 6 \\
\hline
\end{tabular}

Die Exemplifikation der unter den Wortarten am zahlreichsten vorkommenden lexikalischen Einheiten speist sich aus dem substantivischen Wortschatz und soll vor allem die Realisierung weiterer notfallmedizinischen Sprachkurse des Polnischen für berufliche Zwecke erleichtern. In dieser Kategorie werden folgende Einheiten von den Teilnehmenden aufgeführt: badanie (Untersuchung), ból (Schmerz), brzuch (Bauch), głos (Stimme), głowa (Kopf), klatka piersiowa (Brustkorb), kolka (Kolik), lek (Medikament), nadbrzusze (Oberbauch), norma (Norm), pan (Herr / Sie), papierosy (Zigaretten), podbrzusze (Unterbauch), rana kasana (Bisswunde), skala (Skala), szpital (Krankenhaus).

Verben stellen gleichermaßen ein wichtiges Element des Wortschatzes dar und somit wurden folgende Formen ausgemacht: boli, czuje, jadt, kłuć, opisać, promieniuje. Um Sprachunterricht für notfallmedizinische Kommunikation erfolgreich zu gestalten, werden die Verben in Infinitivform erneut aufgelistet, d.h. von den Teilnehmenden wurden folgende Einheiten verzeichnet: boleć (schmerzen, wehtun), czuć (fühlen), jeść (essen), kłuć (stechen), opisać (beschreiben), promieniować (ausstrahlen).

\subsection{Wortschatz im Themenbereich „Patientenübergabe“}

Im Rahmen des thematischen Bereichs „Patientenübergabe“ wurden 43 sich nicht wiederholende Einheiten verzeichnet. Die lexikalischen Einheiten, die sich in der Frequenzanalyse am häufigsten wiederholen, werden in der Tabelle aufgelistet.

Tabelle 5. Am häufigsten gennannte lexikalische Einheiten im Themenbereich „Patientenübergabe

\begin{tabular}{|l|c|}
\hline \multicolumn{1}{|c|}{ Lexem } & Häufigkeit \\
\hline puls (Puls) & 12 \\
\hline saturacja (Sauerstoffsättigung) & 12 \\
\hline drogi oddechowe (Atemwege) & 10 \\
\hline
\end{tabular}


In allen vier Gruppen werden von den Teilnehmenden die lexikalischen Einheiten puls und saturacja genannt. Wiederum drogi oddechowe werden von den Teilnehmenden in drei Gruppen verzeichnet.

In diesem Themenbereich setzt sich der Wortschatz anhand von Wortarten wie folgt zusammen: Die Mehrheit machen Substantive (77\%) aus, gefolgt von Adjektiven (9\%). Verben kommen nur zu 7\% vor. Eine detaillierte Zusammensetzung des Wortschatzes zum Thema "Patientenübergabe“ ist der Tabelle zu entnehmen.

Tabelle 6. Wortschatz zum Thema „Patientenübergabe“ nach Wortarten $(n=43)$

\begin{tabular}{|c|c|c|c|c|c|c|}
\hline Wortart & Adjektiv & Adverb & Pronomen & Präposition & Substantiv & Verb \\
\hline Anzahl & 4 & 1 & 1 & 1 & 33 & 3 \\
\hline
\end{tabular}

Ähnlich wie in den Themenbereichen „Anamnese“ und „Schmerzuntersuchung" soll hier die Exemplifikation der unter den Wortarten am zahlreichsten vorkommenden lexikalischen Einheiten vor allem die Konzeption und Realisierung weiterer notfallmedizinischen Sprachkurse des Polnischen für berufliche Zwecke vereinfachen. Es wurden von den Teilnehmenden folgende substantivische lexikalische Einheiten genannt: alergia (Allergie), anamneza (Anamnese), badanie (Untersuchung), ból (Schmerz), choroba (Krankheit), cukier (Zucker), częstość (Frequenz), drogi oddechowe (Atemwege), glukoza (Glukose), lat (Jahre), leki (Medikamente), objętość (Volumen), obrażenia (Verletzung), oddech (Atmung), otarcia (Abschürfungen), pacjent (Patient), puls (Puls), rana (Wunde), saturacja (Sauerstoffsätigung), serce (Herz), skala (Skala), szmer (Atmungsgeräusch), temperatura (Temeperatur), tętno (Puls), tlen (Sauerstoff), uraz (Trauma), wypadek (Unfall), zawał (Herzinfarkt), źrenica (Pupille). Zu der Auflistung gehören auch Abkürzungen: ABCDE, ATMIST, EKG, GCS.

Des Weiteren wurden von den Teilnehmenden folgende Verbformen genannt: boli, choruje, podaliśmy. Um Sprachunterricht für notfallmedizinische Kommunikation durchzuführen, werden die Verben in Infinitivform erneut aufgelistet, d.h. von den Teilnehmenden wurden folgende Einheiten genannt: boleć (schmerzen, wehtun), chorować (krank sein), podawać (verabreichen).

\subsection{Grundwortschatz für die Notfallkommunikation}

Bei der Analyse des Wortschatzes der drei festgelegten Themenbereiche ist aufgefallen, dass sich einige lexikalische Einheiten in mindestens zwei Bereichen wiederholen. Dies verleitet dazu, von einer Art von Grundwortschatz 
für die Notfallkommunikation zu sprechen und diesen festzuhalten, um die Konzipierung und die Realisierung von Sprachkursen zu unterstützen. Folgende lexikalische Einheiten wurden als substantivischer Grundwortschatz dank den Teilnehmenden ausgemacht: alergia (Allergie), badanie (Untersuchung), ból (Schmerz), choroba (Krankheit), głowa (Kopf), klatka piersiowa (Brustkorb), lat (Jahre), leki (Medikamente), pan (Herr / Sie), rana (Wunde), serce (Herz), skala (Skala), szpital (Krankenhaus), temperatura (Temperatur), zawat (Herzinfarkt). Dieser Grundwortschatz lässt sich in eine größere Art thematische Grundwortschatzliste zusammenfassen, die Körperteile, Wundarten, Medikamente, Symptome, Allergien und Krankheitsbilder notfallmedizinischer Kommunikation. Auf Grund derer könnten kommunikative Aufgaben entwickelt werden, die im Kern dann auf den Rettungsschemata basieren würden. Der gesammelte Wortschatz ist zur Entwicklung eines Lehrprogramms dahingehend nützlich, dass zu einem eine Eingrenzung des Stoffs möglich ist, was für basale notfallmedizinische Kommunikation von besonderer Relevanz ist, zu anderem wäre dies prinzipiell wichtig, um Erfordernisse an die Teilnehmenden anzupassen und unter den Fremdsprachendidaktikern eine Standardisierung im Sinne eines Referenzrahmens für notfallmedizinische Kommunikation vorzunehmen.

Auch die Verben lassen sich zu einem Grundwortschatz zusammenfassen. Die Auflistung ist kurz, sie umfasst zwei Verben: boleć (schmerzen, wehtun) und jeść (essen).

\subsection{Hören und Sprechen als Fertigkeiten bei der Notfallkommunikation}

Bei fremdsprachlichen Kursen sind Wortschatzkenntnisse üblicherweise an eine fremdsprachliche Konzeption gekoppelt, die ihren Inhalt anhand des Gemeinsamen Europäischen Referenzrahmens (GER) ausrichtet. Ähnlich ist es bei diesem besonderen Sprachkurs, auch wenn für das Polnische kein Referenzrahmen für Fachsprachenunterricht vorliegt.

Ein erster Entwurf eines solchen Referenzrahmen wurde bereits im Rahmen von methodischen Überlegungen erstellt (Lisek 2019: 23) und im Laufe der Erprobung der Lehrmaterialien analysiert, reflektiert und ergänzt. Des Weiteren hat die hiesige Untersuchung gezeigt, welche lexikalischen Einheiten bei welchen thematischen Schwerpunkten zu erwarten sind. Durch die Frequenzanalyse des gesammelten Wortschatzes konnte darüber hinaus gezeigt werden, welche Relevanz die Rettungskräfte einzelnen Sprechhandlungen, die sich in dem Wortschatz materialisieren, zuschreiben. 
Betrachtet man die Erkenntnisse der Untersuchung, könnte die Beschreibung des Referenzrahmens für Hören und Sprechen (dabei spielt der Wortschatz eine wichtige Rolle) in Anlehnung an den durchgeführten Sprachkurs wie folgt aussehen:

Tabelle 7. Beschreibung ausgewählter Fertigkeiten auf dem basalen Niveau des Polnischen als

Fremdsprache im Rahmen der medizinischen Fachkommunikation für den Rettungsdienst

\begin{tabular}{|l|l|}
\hline Hören & $\begin{array}{l}\text { Kursteilnehmende können vertraute Wörter und elliptische (nominale und / oder } \\
\text { verbale) Äußerungen oder ganz einfache Sätze verstehen, die sich auf die Patienten } \\
\text { (Körperteile, Symptome, Krankheiten), sie selbst, das Rettungsteam oder auf konk- } \\
\text { rete Gegenstände im Krankenhaus, im Rettungswagen oder (sehr eingeschränkt) am } \\
\text { Einsatzort (z. B. bei der Zugangslegung oder Reanimation) beziehen, vorausgesetzt } \\
\text { es wird auf Polnisch langsam und deutlich genug gesprochen. }\end{array}$ \\
\hline Sprechen & $\begin{array}{l}\text { Kursteilnehmende können sich auf eine sehr einfache Art (mittels elliptischer nomi- } \\
\text { naler oder verbaler Äußerungen oder einfacher Sätze) verständigen, wenn ihre } \\
\text { Gesprächspartner bereit sind, sich langsam zu artikulieren oder Gesagtes zu wieder- } \\
\text { holen. Kursteilnehmende können die für die notfallmedizinische Situation typischen } \\
\text { und einfachen Fragenhandlungen realisieren (z. B. Fragen oder Aufforderungen beim } \\
\text { Anamnesegespräch im Rahmen der Schemata: SAMPLE(R) und OPQRST und beant- } \\
\text { worten (z. B. bei der Patientenübergabe im Rahmen des Schemas ABCDE, SBAR oder } \\
\text { ATMIST), sofern es sich um vertraute Themen (z. B. Körperteile, Krankheitssymp- } \\
\text { tome, Medikamentenverabreichung, Rettungswagenausstattung) handelt. }\end{array}$ \\
\hline
\end{tabular}

\section{ZUSAMMENFASSENDE BEMERKUNGEN UND AUSBLICK}

Der hier gesammelte Wortschatz, der mittels einer Frequenzanalyse überprüft wurde, zeigt, dass im Vordergrund des analysierten Unterrichts abhängig von Themenbereichen, beispielsweise "notfallmedizinische Anamnese“, ból (Schmerz) und leki (Medikamente) stehen.

Auch symptomy (Symptome) und duszności (Atemnot) gehören, laut Teilnehmenden, dem häufig vorkommenden Wortschatz an. Die zeitliche und räumliche Dimension in der notfallmedizinischen Kommunikation können, so die Teilnehmenden, mit den Fragepronomen kiedy (wann) und gdzie (wo) realisiert werden. Die Fragepronomen werden hier ergänzt mit einem weiteren Pronomen - od (seit), das in Verbindung mit kiedy, die Frage od kiedy (seit wann) ergibt. Zu der sich am häufigsten bei der Patientenübergabe wiederholenden VitalparameterLexik gehören saturacja (Sauerstoffsättigung) und puls (Puls).

Dank des gesammelten Wortschatzes wurde nicht nur die Themenrelevanz überprüft, sondern auch eine Grundlage für ein fachsprachliches zielgerichtetes Kursangebot für Polnisch für Rettungskräfte geschaffen, in Form eines zusammengefassten substantivischverbaler Grundwortschatzes. 
Die hier gewonnenen Erkenntnisse könnten in die Beschreibung der Fertigkeiten Hören und Sprechen einfließen, die sich an GER orientiert. Dies wäre ein praktischer und produktiver Weg die Ergebnisse der vorliegenden Untersuchung didaktisch aufzubereiten und aufzuwerten.

\section{LITERATURVERZEICHNIS}

Albert, R. / Marx, N. (2016). Empirisches Arbeiten in Linguistik und Sprachlehrforschung. Anleitung zu quantitativen Studien von der Planung bis zum Forschungsbericht. Tübingen: Narr.

Chłopicka-Wielgos, M. / Pukas-Palimąka, D. / Turek-Fornelska, K. (2015). Co Panu dolega? Podręcznik rekomendowany przez Centrum Języka i Kultury Polskiej w Świecie Uniwersytetu Jagiellońskiego do nauczania cudzoziemców języka polskiego na studiach medycznych. Kraków: FWKiJP im. Mikołaja Reja.

Fluck, H.R. (1992). Didaktik der Fachsprachen: Aufgaben und Arbeitsfelder, Konzepte und Perspektiven im Sprachbereich Deutsch. Tübingen: Narr.

Gajewska, E. (2015). Poziomy biegłości a nauczanie języków specjalistycznych. In: S. Piotrowski (Hrsg.), O nauczaniu i uczeniu się języka obcego dla potrzeb zawodowych (S. 10-21). Lublin: Werset.

Gębal, P.E. / Kołsut, S. (2019). Zur Berufsorientierung des Fachsprachenunterrichts im mitteleuropäischen Kontext. Von den Arbeitsmarkterwartungen zu den berufsspezifischen Sprachkompetenzprofilen. Studia Neofilologiczne, XV, 61-71. http:/ / dx.doi.org/10.16926/sn.2019.15.05 [Zugriff am: 25.10.2020].

Gębka-Wolak, M. (2011). Przygotowanie lektorów do nauczania języka specjalistycznego. In: K. Pluskota / K. Taczyńska (Hrsg.), Nowe perspektywy w nauczaniu języka polskiego jako obcego II (S. 303-320). Toruń: Wydawnictwo Naukowe UMK.

Gębka-Wolak, M. (2016). Gramatyka w nauczaniu języka polskiego do celów specjalistycznych. In: E. Baglajewska-Miglus / T. Vogel (Hrsg.), Fachsprachenunterricht: Lernen und Lehren am Beispiel des Polnischen als Fremdsprache / Nauczanie języka specjalistycznego: Nauczanie i uczenie się na przyktadzie języka polskiego jako obcego (S. 9-24). Aachen: Shaker Verlag.

Grucza, S. (2010). Sprache(n) - Fachsprache(n) - Fachsprachendidaktik. Studia Germanica Gedanensia, 22, 31-46.

Hryniewicz, J. / Lisek, G. (2019). Nauka języka sąsiada: od kształcenia przedszkolnego do zawodowego. Rozwiązania dydaktyczne i organizacyjne na Pomorzu Przednim. In: K. Zioło-Pużuk (Hrsg.), Panorama glottodydaktyki polonistycznej. Wyzwania, pytania, kierunki (S. 149-162). Warszawa: Wydawnictwo UKSW.

Kósa, G. (2019). Lexikalische Kompetenz im schulischen Fremdsprachenunterricht. Werkstatt, 14, 30-42.

Lisek, G. (2018). Wie motiviere ich Teilnehmer bei einem ELearningFachsprachenkurs? Reflexionen zum ELearning für einen PolnischFachsprachenkurs für Rettungskräfte. In: E. Baglajewska-Miglus / T. Vogel (Hrsg.), Polnisch als Fremd und Zweitsprache, Band 4: Fachsprache Polnisch Sprache mit Zukunft. Specjalistyczny język polski - język z przyszłościa (S. 106-112). Aachen: Shaker Verlag.

Lisek, G. (2019). Fachkommunikation und regionale Vernetzung am Beispiel des Projekts Integrierter grenzüberschreitender Rettungsdienst Pomerania / Brandenburg (InGRiP). Greifswalder Beiträge zur Hochschullehre. Perspektiven der Lehre im 21. Jahrhundert, 10, 21-32. 
Lisek, G. (2020). Notfallmedizinische Kommunikation im Unterricht Polnisch als Fremdsprache. Analyse von Gesprächsverhalten deutschsprachiger Rettungskräfte. Glottodidactica. An International Journal of Applied Linguistics, XLVII (2), 171-195.

Majewska-Wójcik, A. / Račáková, A. / Smoleń-Wawrzusiszyn, M. / Olchowa, G. (2016). Dwujęzyczny podręcznik komunikacji jezykowej dla stużb ratowniczych pogranicza polskostowackiego. Lublin: Wydawnictwo KUL.

Roche, J. (2013). Fremdsprachenerwerb - Fremdsprachendidaktik. Tübingen, Basel: Francke.

Searle, J.R. (1997). Sprechakte: ein sprachphilosophischer Essay. Frankfurt (a. M.): Suhrkamp.

Seretny, A. (2010). Rozwijanie kompetencji leksykalnej uczących się poprzez obcowanie z tekstem. Acta Universitatis Lodziensis. Ksztatcenie Polonistyczne Cudzoziemców, 17, 547-579.

Seretny, A. (2017). Leksyka w nauczaniu języka specjalistycznego - potrzeby akademickie a potrzeby zawodowe. Acta Universitatis Lodziensis. Kształcenie Polonistyczne Cudzoziemców, 24, 149-166.

Stemmler, J. / Hecker, U. (2017). Notfallkommando Kommunikation in Notfallsituationen für Gesundheitsberufe. Berlin, Heidelberg: Springer.

Targońska, J. (2011). Lexikalische Kompetenz - ein Plädoyer für eine breitere Auffassung des Begriffs. Glottodidactica. An International Journal of Applied Linguistics, XXXVII, 117-127.

Targońska, J. (2014). Lexikalische Strategien der Germanistikstudierenden zu ihrem Studienbeginn. Lingwistyka Stosowana, 9, 171-200.

Received: 20.11.2020; revised: 11.03 .2021

\section{GRZEGORZ LISEK}

Universität Greifswald

grzegorz.lisek@unigreifswald.de

ORCID: 0000000316133855 
